\title{
Neumopericardio a tensión en trauma cerrado de tórax por efecto Macklin. Reporte de caso
}

\author{
Alejandro Gutiérrez-Ospina,凶 Guillermo Careaga-Reyna, Carlos Alberto Lezama-Urtecho, \\ Luis Alberto Quispe-Fernández.
}

UMAE Hospital General "Dr. Gaudencio González Garza” del Centro Médico Nacional "La Raza"

Trabajo recibido: 29-XI-2016; Aceptado: 12-XII-2016

\begin{abstract}
RESUMEN. El neumopericardio es la presencia de aire en el espacio pericárdico, siendo una entidad infrecuente de causa multifactorial. El diagnóstico se hace por estudios de imagen. Por lo general es autolimitado, pero potencialmente mortal si evoluciona a taponamiento cardíaco. Aunque hay pocos casos reportados en la literatura y uno postraumático, creemos que el efecto Macklin como causa de neumopericardio sin neumomediastino está subdiagnosticado y podría estar presente en los casos espontáneos. Se describe el caso de una paciente con neumopericardio a tensión postraumático como resultado del efecto Macklin con tratamiento toracoscópico exitoso.
\end{abstract}

Palabras clave: Efecto Macklin, neumopericardio, taponamiento cardíaco, toracoscopia, trauma cerrado de tórax.

ABSTRACT. Pneumopericardium is the presence of air inside the pericardial cavity, a rare entity which has multifactorial etiology. It is diagnosed through imaging studies. It's usually a self-limited disease but potentially mortal if it evolves to a cardiac tamponade condition. Even though there are only a few reported cases in scientific literature and only one of which are post traumatic; we believe that there's an underdiagnosis of the Macklin effect a cause of pneumopericardium without pneumomediastinum, and that it could also be present in spontaneous cases. We describe a posttraumatic tension pneumopericardium case as a result of the Macklin effect, treated successfully with thoracoscopy.

Key words: Macklin effect, pneumopericardium, cardiac taponade, thoracoscopy, blunt chest trauma.

\section{INTRODUCCIÓN}

El neumopericardio es la presencia de aire en la cavidad pericárdica. Es una entidad clínica muy poco frecuente, pero potencialmente mortal ya que puede evolucionar a taponamiento cardíaco (TC)., ${ }^{1,2}$ En adultos la etiología más frecuente es la iatrogénica como complicación de procedimientos invasivos, como son: la canalización de vías centrales, pericardiocentesis o ventilación mecánica, entre otras. ${ }^{1,2}$ También puede vincularse a crisis de asma, cáncer pulmonar o como consecuencia de trauma cerrado de tórax (TCT) o penetrante de tórax generando, además, neumotórax, neumoperitoneo y/o neumomediastino. ${ }^{3}$ En ocasiones excepcionales, es consecuencia de mecanismos que generan ruptura alveolar, disección de las vainas broncovasculares y diseminación del enfisema intersticial pulmonar hacia el mediastino, esto último se conoce como efecto Macklin (EM). ${ }^{4}$ El diagnóstico se hace por radiografía simple o tomografía de tórax (TAC).$^{1-4}$ Por lo general, es autolimitado y no requiere tratamiento específico.
Sin embargo, es necesaria una vigilancia continua en una Unidad de Cuidados Intermedios, por el riesgo de evolucionar a neumopericardio a tensión (NT) con TC requiriendo drenaje pericárdico inmediato. ${ }^{4,5}$

\section{CASO CLÍNICO}

Paciente femenino de 70 años de edad con antecedente de diabetes mellitus tipo 2 (DMT2), hipertensión arterial sistémica, enfermedad pulmonar obstructiva crónica (EPOC) y hernia hiatal manejada hace 15 años con funduplicatura de Nissen abierta. Presenta trauma en hombro izquierdo y región posterior de hemitórax izquierdo por caída de un metro de altura, refiriendo dolor epigástrico con irradiación a tórax anterior y dolor en hombro izquierdo, motivo por el que consulta a hospital de segundo nivel 24 horas después del traumatismo. Recibió manejo conservador con analgésicos pero hay persistencia de los síntomas, agregándose intolerancia a la vía oral. Se realizan estudios de imagen y es enviada a valoración por cirugía cardiotorácica por 
sospecha de neumomediastino. A su llegada al tercer nivel de atención, presenta descontrol metabólico, deshidratación con elevación de azoados sin compromiso hemodinámico o respiratorio, se da tratamiento médico para DMT2 descompensada y lesión renal aguda AKIN III, pasando a manejo por terapia intermedia en el servicio de Urgencias.

La radiografía de tórax mostró una banda radiotransparente paracardíaca sugestiva de neumopericardio, sin encontrar neumotórax o derrame pleural (figura 1).

TAC toracoabdominal en la que se observó neumopericardio, bulas bilaterales, aire perivascular y peribronquial, sin neumomediastino o neumotórax (figura 2). Se realizó esofagograma en donde se descartó perforación esofágica y con funduplicatura permeable. Ecocardiograma transtorácico demostró aire intrapericárdico con colapso leve de aurícula derecha con fracción de eyección del ventrículo izquierdo y derecho conservada. A las pocas horas, la paciente presentó datos de TC diagnosticado por inestabilidad hemodinámica demostrada por hipotensión sostenida, a pesar de manejo con aminas vasoactivas y alteraciones del ritmo (alternancia eléctrica). Se pasó a quirófano urgente para drenaje pericárdico toracoscópico (figura 3 ). En el reporte quirúrgico se describe aire intrapericárdico con escaso derrame pericárdico de $100 \mathrm{~mL}$ seroso y fibrina intrapericárdica. En el posquirúrgico inmediato hay mejoría evidente de la hemodinámica de la paciente y pasó a Terapia de Cuidados Intensivos posquirúrgicos cardiovasculares, donde permaneció 24 horas sin requerir manejo avanzado de la vía aérea ni

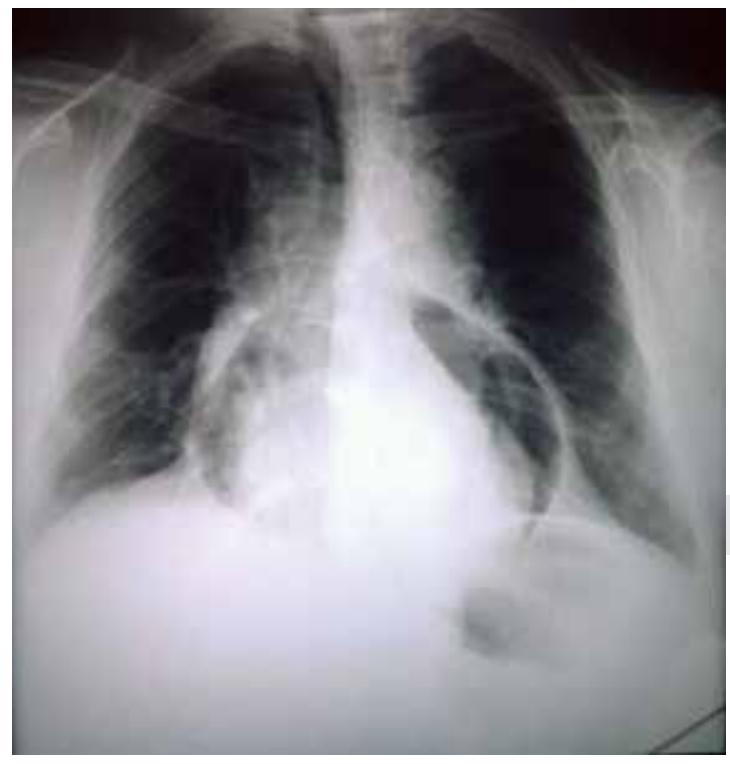

Figura 1. Banda radiotransparente paracardíaca secundaria a aire intrapericárdico. apoyo aminérgico. Los resultados de los cultivos en las muestras tomadas en evento quirúrgico aíslan en tejido pericárdico y líquido pericárdico Staphylococcus sciuri (contaminación), sin requerir manejo antimicrobiano específico. Pasó a sala de hospitalización de cirugía cardiotorácica, en donde evolucionó adecuadamente sin salida de líquido por la sonda de drenaje pericárdico, la cual se retira a las 72 horas con control radiográfico que no muestra alteraciones. Se da de alta a su domicilio a los 7 días posteriores al evento quirúrgico.

\section{DISCUSIÓN}

El neumopericardio es la presencia de gas en el saco pericárdico. Generalmente es de comportamiento benigno sin requerir tratamiento específico. ${ }^{6}$ Sin embargo, en informes documentados por Capizzi et al., ${ }^{7}$ el $37 \%$

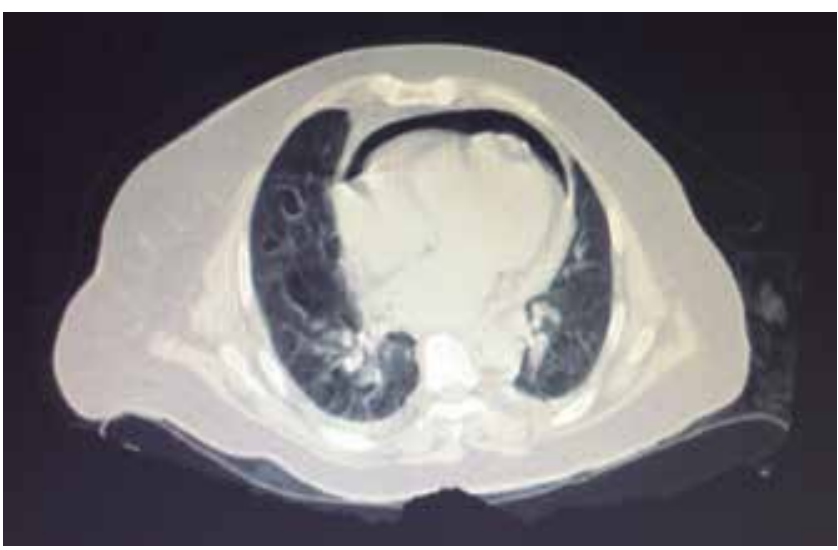

Figura 2. Se aprecia neumopericardio, bulas y tanto aire perivascular como peribronquial disecando estos espacios.

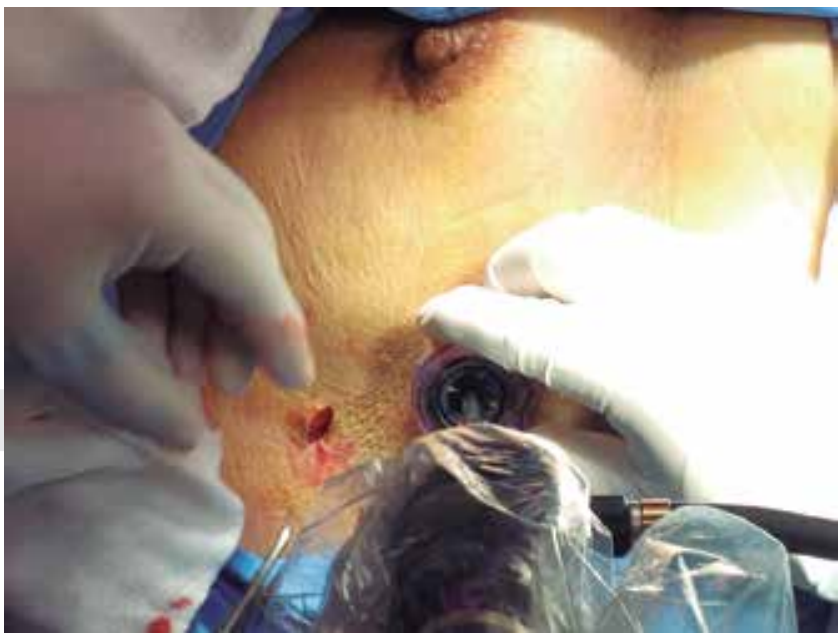

Figura 3. Abordaje toracoscópico derecho para drenaje pericárdico. 
de los pacientes con neumopericardio después de un TCT presentó NT. EI NT es una causa inusual de compromiso hemodinámico en el TCT. Otras causas más comunes de hipotensión, como choque hemorrágico o neumotórax a tensión deben considerarse primero. ${ }^{8,9}$ Se puede asociar con una tasa de mortalidad hasta del $57 \%$ al ser potencial causa de TC además del politraumatismo asociado, teniendo en cuenta que se encuentra por lo regular en accidentes de alta energía como lo reportan Marques et al. ${ }^{10}$

Clásicamente, la presencia de aire en el pericardio se produce después del trauma penetrante de tórax. Sólo 32 casos de neumopericardio debido a diferentes causas tras un TCT se han reportado en la literatura. ${ }^{11}$

Hay diferentes mecanismos propuestos para el desarrollo del neumopericardio en el TCT. Puede ser causado por un aumento abrupto de la presión intratorácica con la consecuente ruptura alveolar. En este caso, la gravedad del trauma debe ser lo suficientemente grande como para causar desgarro pleural, pericárdico y bronquial que ocasiona neumotórax, neumomediastino y neumopericardio. En este contexto, las comunicaciones pleuropericárdicas congénitas podrían explicar la aparición de neumopericardio en casos contados. ${ }^{12,13}$ Finalmente, se encuentra la ruptura alveolar con disección de las vainas broncovasculares y diseminación del enfisema intersticial pulmonar hacia el hilio y el pericardio, éste es el efecto conocido como Macklin. ${ }^{14,15}$ Descrito en 1939 por Macklin, quien reportó el proceso fisiopatológico en el que la ruptura alveolar es la responsable del neumomediastino. ${ }^{16}$

Consideramos que la causa del neumopericardio en el caso que reportamos fue secundaria al incremento de la presión intraalveolar, provocando la ruptura de alvéolos con la consecuente fuga de aire, alcanzando el pericardio parietal (efecto Macklin). Éste sería el segundo caso reportado en la literatura como causa de neumopericardio por EM en TCT y, hasta el momento, el único sin neumotórax o neumomediastino concomitante. El EM está ligado pero no limitado a contusión torácica. También se puede presentar con otras patologías como síndrome de dificultad respiratoria, crisis y estado asmático, ventilación mecánica con presión positiva y las maniobras de Valsalva. ${ }^{17,18}$

El diagnóstico de neumopericardio puede realizarse con una Rx de tórax simple que suele mostrar una banda radiotransparente paracardíaca en un paciente con dolor torácico de tipo pleuropericárdico. ${ }^{19}$ En la TAC de tórax se observa el aire intrapericárdico y permite diferenciarlo del neumomediastino, ya que en el neumopericardio el aire cambia de posición al adoptar el decúbito supino. ${ }^{20}$
La TAC en nuestro caso fue de vital importancia para asociar la causa del neumopericardio con el EM, pues en ella observamos neumopericardio asociado a aire perivascular y peribronquial, como se describe en los diferentes reportes en los que se cita al EM.

Casi siempre, el neumopericardio es autolimitado y requiere manejo expectante en la mayoría de los casos. ${ }^{21}$ Sin embargo, como en nuestro caso, si el proceso evoluciona a TC, el drenaje pericárdico inmediato es necesario, bien sea por ventana pericárdica abierta, pericardiocentesis o drenaje vía toracoscopia como en el caso reportado en este artículo. ${ }^{22}$ El diagnóstico clínico y la vigilancia continua del paciente son básicos para el diagnóstico de TC, y detectar los signos a tiempo es una maniobra salvavidas para el tratamiento oportuno. Los signos de TC son: pulso paradójico, taquicardia, hipotensión, alternancia eléctrica en el electrocardiograma o monitor continuo cardíaco, aumento de la presión venosa central (plétora yugular) y ruidos cardíacos velados. ${ }^{23}$

\section{CONCLUSIONES}

Son pocos los casos reportados en la literatura que adjudican al EM como causa de neumopericardio y tan sólo un caso reportado secundario a TCT y EM asociado. Por ello, creemos que el EM como causa de neumopericardio sin neumomediastino o neumotórax está subdiagnosticado y podría estar presente en muchos de los casos espontáneos.

El neumopericardio es autolimitado en la mayoría de los casos. No obstante, el TC por NT ordena descompresión urgente y debe ser adecuadamente descartado para no confundirlo con otras entidades que amenazan la vida presente en el TCT, como son: la contusión cardíaca, pulmón de choque, hemorragia, hemotórax masivo o neumotórax a tensión. Este caso demuestra el drenaje rápido y eficaz vía toracoscópica.

\section{REFERENCIAS}

1. Cafaro G, Viale M, Leandro I, Sánchez R, Zivano D, Monetti E. Neumopericardio. Rev Fed Arg Cardiol 2011;40(3).

2. Barquero-Romero J, Izquierdo-Hidalgo J, Maciá-Botejara E, Arrobas-Vacas J, Pérez-Miranda M. Neumopericardio espontáneo en un paciente con neumonía de la comunidad. Rev Esp Cardiol 2005;58(2):227-229.

3. Ladurner R, Qvick LM, Hohenbleicher F, Hallfeldt KK, Mutschler W, Mussack T. Pneumopericardium in blunt chest trauma after high-speed motor vehicle accidents. Am J Emerg Med 2005;23(1):83-86.

4. Martín-Menjívar E, Vázquez-Minero JC, FerreroBalado E, Domínguez A. Efecto Macklin como causa 
de neumomediastino posterior a contusión torácica. Reporte de un caso. TRAUMA 2007;10(2):58-61.

5. Barbetakis N, Asteriou C, Kleontas A. Pathophysiology of pneumopericardium after blunt thoracic trauma. Ann Thorac Surg 2011;92(5):1936. doi: 10.1016/j. athoracsur.2011.01.090.

6. Gould JC, Schurr MA. Tension pneumopericardium after blunt chest trauma. Ann Thorac Surg 2001;72(5):1728-1730.

7. O'Connor JV, Scalea TM. Tension pneumopericardium after blunt thoracic Trauma. Ann Thorac Surg 2010;90(5):1713. doi: 10.1016/j.athoracsur.2010.01.086.

8. Zakynthinos E, Karetsi E, Diakaki C. Pneumopericardium after blunt chest trauma: Mechanical ventilation with positive pressure must be avoided. 2008;124(1):e8-e10.

9. Kallel N, Beloeil H, Geffroy A, Albaladejo P, Marty J. Post-traumatic tension pneumothorax and pneumopericardium in spontaneous ventilation. Ann Fr Anesth Réanim 2004;23(4):364-366.

10. Rolim-Marques AF, Lopes LH, Martins Mdos S, Carmona CV, Fraga GP, Hirano ES. Tension pneumopericardium in blunt thoracic trauma. Int J Surg Case Rep 2016;24:188190. doi: 10.1016/j.ijscr.2016.04.052.

11. Roth TC, Schmid RA. Pneumopericardium after blunt chest trauma: asign of severe injury? J Thorac Cardiovasc Surg 2002;124(3):630-631.

12. Chassagnon G, Favelle O, Derogis V, Cottier JP. Spontaneous pneumomediastinum due to the Macklin effect: less is more. Intern Emerg Med 2015;10(6):759761. doi: 10.1007/s11739-015-1229-1.

13. Nachi $\mathrm{S}, \mathrm{Okada} \mathrm{H}$, Kato $\mathrm{H}$, et al. Simple pneumopericardium due to blunt trauma progressing to tension pneumopericardium during transportation. Am J Emerg Med 2016;34(5):933.e3-5. doi: 10.1016/j.ajem.2015.09.028.

14. Carzolio-Trujillo HA, Navarro-Tovar F, Padilla-Gómez $\mathrm{Cl}$, Hernández-Martínez IA, Herrera-Enríquez J. Trauma contuso de tórax con neumomediastino y neumoperitoneo secundario a efecto Macklin. Reporte de un caso. Cirugía y Cirujanos 2016;84(5):409-414). doi: 10.1016/j.circir.2015.05.055

15. El-Shammaa EN, Martin DR. Spontaneous pyopneumopericardium. Am J Emerg Med 1999;17(3): 245-247.
16. Sekhar A, Raheja $\mathrm{P}$, Ikram S. The Macklin effect causing a pneumopericardium. J Cardiovasc Med (Hagerstown) 2012;13(8):527-528. doi: 10.2459/ JCM.0b013e328356bc37.

17. Agarwal MP, Giri S, Jain R, Sharma V. Spontaneous pneumopericardium in acute asthma. Int J Emerg Med 2010;3(2):141. doi: 10.1007/s12245-010-0158-z.

18. Tsai WC, Lin LJ, Chen JH, Wu MH. Afebrile spontaneous pneumopyopericardium. Int J Cardiol 1996;54(1):69-72.

19. Wu M, He X, Yang G. Spontaneous tension hydropneumopericardium complicating serofibrinous pericarditis. Eur J Cardiothorac Surg. 2006; 29(3):422424.

20. Chopra V, Garg N, Mrigpuri P. Spontaneous pneumopericardium an unusual complication in a patient of HIV and pulmonary tuberculosis. Lung India. 2013; 30(2):148-150. doi: 10.4103/0970-2113.110425.

21. Castagno D, Dalzell JR, Jackson CE, Neisius U, Cormack A, Gaita $F$. The heart in an airbag: spontaneous pneumopericardium in a young lady. J Cardiovasc Med (Hagerstown) 2012;13(12):833-835. doi: 10.2459/ JCM.0b013e32834ae6ac.

22. Macgoey P, Schamm M, Degiannis E. Tension pneumopericardium: case report. Ulus Travma Acil Cerrahi Derg. 2010;16(5):477-479.

23. Konijn AJ, Egbers PH, Kuiper MA. Pneumopericardium should be considered with electrocardiogram changes after blunt chest trauma: a case report. J Med Case Rep 2008;2:100. doi: 10.1186/1752-1947-2-100.

\section{$\triangle$ Correspondencia:}

Dr. Alejandro Gutiérrez Ospina

HG CMN "La Raza", tercer piso. Avenida de las

Jacarandas y Vallejo sin número $(\mathrm{s} / \mathrm{n})$,

C.P. 02990, Colonia La Raza,

Del. Azcapotzalco, Ciudad de México.

Teléfono: 57245900, ext. 23425.

Correo electrónico: alemed3@hotmail.com

Los autores declaran no tener conflicto de intereses. 